\title{
Comparison between macaques' and humans' kinematics of prehension: the role of morphological differences and control mechanisms
}

\author{
Marianne I. Christel ${ }^{\mathrm{a}, *}$, Aude Billard ${ }^{\mathrm{b}}$ \\ ${ }^{a}$ Institut für Spezielle Zoologie und Evolutionsbiologie, Friedrich-Schiller-Universitaet, Erbertstrasse 1, D-07743 Jena, Germany \\ ${ }^{\mathrm{b}}$ Computer Science Department, University of Southern California, Hedco Neuroscience Building, 3641 Watt Way, Los Angeles, \\ CA 90089-2520, USA
}

Received 7 March 2001; received in revised form 22 August 2001; accepted 22 August 2001

\begin{abstract}
Reaching and grasping has been widely studied in both macaques and humans, mainly with the aim of finding similar patterns of behavior in the two species. Little attention has yet been given to how morphological and behavioral differences between the two species might affect the kinematics of the movement. In this study, we present a careful analysis of the similarities and differences between humans' and macaques' prehension movements and discuss these with respect to both the control system and the biomechanics of the arm. Five humans and five macaques performed the same task, namely grasping small feeding objects using a precision grip. Macaques were observed in unconstrained conditions, free to adjust their body posture. The behavioral protocol for macaques revealed a postural preference for sitting and keeping the elbow slightly flexed when applying a precision grip. In agreement with the literature, kinematics revealed general features of movement common to both humans and macaques. However, within a similar timeframe, macaques produced steeper and wider excursion of the elbow and of the wrist, smaller abduction of the shoulder joint and larger displacement of the torso than humans did. The three-joint limb revealed stronger irregularities for the macaques. We hypothesize that the larger kinematic irregularities and the specific elbow-shoulder posture in macaques result in part from an effort of the control system to compensate for different biomechanical constraints, namely for limited shoulder-joint excursion, in order to achieve a similar range of comfort of motion. Finally, we briefly consider the influence of primitive neural circuits responsible for arm motion during locomotion and speculated on their influence on the control of reaching in macaques. C 2002 Elsevier Science B.V. All rights reserved.
\end{abstract}

Keywords: Natural prehension; Reach-to-grasp kinematics; Shoulder and arm displacements; Morphological constraints; Species differences; Macaques and humans

\section{Introduction}

The study of reaching and grasping behavior in non-human primates has become a popular paradigm to compare motor planning and control. So far, comparative studies of macaques' and humans' grasping focused on finding similarities across the two species

\footnotetext{
* Corresponding author.

E-mail addresses: mchriste@zedat.fu-berlin.de (M.I. Christel), billard@usc.edu (A. Billard).
}

behaviors, such as to support the use of studies in macaques as a model of the similar mechanisms in humans. However, monkeys use of their upper limbs differs from that of humans. Macaques must switch rapidly from a powerful limb muscle control during locomotion to a fine-tuned one for fast and precise grasping. This distinctive use of upper limbs in macaques puts different constraints on the control system and arm morphology than those in humans. Such differences would be revealed when comparing the two species' kinematics of reach-to-grasp movements. We 
first review evidence for similarities between macaques' and humans' kinematics of arm movement and then review evidence of differences in the two species' biomechanics of the arm.

\section{Species similarities in kinematics}

Current literature agrees on a number of features for reaching movements common to both macaques and humans. The motion of the hand (end-point) in Cartesian space has a bell-shaped velocity profile and follows an approximately straight path $[18,38]$. The latter feature has also been shown in blind subjects [35], suggesting a control independent on visual input. The duration and amplitude of the movement varies with the distance and the degree of difficulty of the task, following a speed vs. accuracy trade-off paradigm defined by Fitts [12]. Both humans $[19,26]$ and macaques [30,34] adapt both the timing of hand preshape and the size of the finger aperture to the object's size and location.

Interestingly, these basic features of prehension kinematics (quasi-straight path of the end-effector, variation of peak velocity with distance to object, and variation of timing and size of the maximum aperture with object size) seem not unique to primates. They were shown in other generic of family, phylogenetically widely separated from primates and, thus, for different effectors. Studies in pigeons [2,20] showed that size, velocity and duration of gape opening, as well as velocity and position of head bending varied monotonically with the size of the seed. Study of paw motion in cats revealed a continuous scaling of stereotyped trajectory profiles (with bell-shape velocity profile) as a function of the target location [22].

Taken together, these invariant features across species and across effectors seem to reflect fundamental mechanisms underlying how the central nervous system (CNS) plans and executes reaching movements. The question of what the CNS computes exactly is, however, still the topic of a large debate. A general view is that goal-directed reaching movements are represented in the CNS as task-specific action units [3], which control the temporal co-ordination of muscle synergies. This approach favors the idea of a feedback loop between the CNS and the spinal cord $[6,15]$. The CNS generates commands, based on muscular feedback, to sets of subcortical neural controllers (central pattern generators (CPGs), responsible for reflexes and primitive locomotion). A general concern is that there is not enough time for proprioceptive feedback to reach the CNS and to affect the motor commands. Thus, it is proposed that rather than relying on sensory feedback, the CNS works in feedforward control. In this approach, the CNS builds 'inverse forward model' of the sensory-motor loop, as a means to predict the expected outcome of a command as well as to estimate the current position and velocity of the moving limbs in the presence of feedback delays (associated with the neural processing of sensory information).

In previous work, Christel and co-workers showed that known differences in the transmission of corticospinal excitation to upper limb motoneurons across primates could also be revealed through behavioral studies $[9,10]$. Macaques have a dense cortico-motoneural system for advanced hand functions [7,14]. A more advanced hand function appears to correlate with a replacement of the propriospinal transmission of corticospinal excitation to cervical motoneurons [23] as it is the case in cats.

The neural organization for fine object prehension in non-human primates and humans seems to result from a complex and intricate interaction between spinal and cortical neural systems, and is yet not completely understood. Moreover, it is yet unclear how the circuits regulating control of arm movements during locomotion are coupled to those regulating reaching. Major findings in that direction come from neurological studies in cats, which revealed some of the neural structures responsible for paw motions during scratching, walking and precise stepping $[17,29,37]$. While the general hierarchical structure of motor control is similar in all vertebrates, the cortico-spinal structures underlying fine control of the elbow, wrist and fingers differs significantly from primates and non-primates, and across primates $[17,23]$. In primates, direct pathways from cortical structures in the motor cortex to pools of moto-neurons provide fine control of the finger digits during object manipulation [7,14]. Primates have greater differentiation of the proximo-distal connections across elbow, wrist and finger extensor muscles than cats, allowing for a more independent use of the wrist and fingers. Similarly to cats, however, spinal structures in non-human primates control co-contraction of the $M$. extensor carpi ulnaris and radialis, which result in a coupling of the wrist and elbow motion [17] and, hence, a good stabilization of the limbs during locomotion.

The above evidence suggests that monkey control of the arms during locomotion might result from evolutionary old structures, located in the spinal cord, and that control of the arm for precise grasping (pinching and manipulation) might result from new cortical structures.

\section{Postural constraints on prehension kinematics in monkeys}

The basic biomechanics (shoulder, elbow, wrist and finger attachment) of non-human primates and human arm is similar. There are, however, a number of impor- 
tant differences in morphology, which presumably affect prehension kinematics. Cheng and Scott [8] investigated several morphometric parameters of the upper limb muscles in Macaca mulatta and Macaca fascicularis. They found that while the extensor/flexor muscle ratios (scaled to body weight) in macaques correspond closely to that of humans, macaques have a larger physiological cross-sectional area of arm muscles, resulting in a greater relative muscle force. The authors concluded that a sort of hypertrophism of macaques arm muscles resulted from their use in generating locomotion.

Study of macaques' arm morphology reveals several other features, which distinguish it from the human arm and might result from the biomechanical constraints created by locomotion. The macaques' shoulder-joint complex differs importantly from the human one. In macaques, the scapula is in a lateral position and is stabilized by a large portion of the M. levator scapulae [21]. Similar to other quadrupeds (e.g. big cats or raccoons), the coracoideum and the acromion in macaques are more largely inclined towards the head of the humerus (Fig. 3). Consequently a number of muscles ( $M$. deltoideus, $M$. coracoacromialis, the short head of the $M$. biceps) have longer levers for anteversion of the arm, while preventing retroversion of the humerus [27]. In several catarrhine primates (like baboons), the head of the humerus is shorter and has a stronger curvature at the dorsal aspect of the capitulum humeri, the processus coracoideus protrudes towards the tuberculum majus [21]. Finally, the olecranon in macaques is longer than in humans; thus the lever of the triceps should be longer [21]. Consequently, the muscle force of the triceps and the torque in elbow joint should be higher. A major difference between humans and nonhuman primates is the existence of $M$. dorsoepitrochlearis, which extends the elbow and adducts the upper arm. This muscle is present in all non-human primates and can be found only in a few humans. This muscle is called the 'climbing muscle' [16] but notes that it might be larger in certain ground-dwelling species than purely arboreal one. This muscle is thought to be an extension of the triceps complex [36].

Fischer and collaborators [11] put forward the idea that function of shoulder complex in primate's locomotion during evolution is reorganized. They use combined methods (cineradiography, EMG, kinematics) [11] to model the scapula and limb function in locomotion of a large number of small to middle-sized quadruped mammals, including small primates (squirrel monkeys). The angle arrangement of this three-segmented forelimb (including the scapula as they argue a four-segmented limb) during locomotion is highly rigid. Varying the fulcrum of the scapula induces limb propulsion and plays an important role for postural change. They showed that squirrel monkeys do not abduct the upper arm independently from scapula [31]. Important function for forelimb reorganization has the $M$. triceps in tuning finely and also stabilizes the upper limbs. These studies provide basic features to discuss constraints by locomotion on primates arm excursion for reach out to grasp.

The observation of important differences between macaques and human shoulder-joint morphology implies that each species has to face different biomechanical constraints on the shoulder joint, which would result in a different range of shoulder motion. There are as yet few studies describing the range of natural shoulder excursion during prehension. Scott and Kalaska [34] showed by joint kinematics that the rotation of the shoulder joint of rhesus monkeys when acting with unconstrained postures is smaller than that of humans (about $20^{\circ}-0-40^{\circ}$ vs. $60^{\circ}-0-95^{\circ}$, zero is the neutral anatomic position). The macaques do not choose to use an abducted arm posture, but could if the task required it [33].

Limited degree of freedom in macaque's shoulderjoint complex might be advantageous during locomotion, as it reduces instability of the forelimbs (which support the body). In other words, the bigger the shoulder displacement, the more unstable the (shoulder-elbow) system and the more torque required to compensate for this instability. Small abduction of the shoulder is, however, less advantageous during reaching. Motion of other limbs (and of the torso) must then be exaggerated in order to compensate for the missing degrees of freedom of the shoulder. Corroborating such a hypothesis, previous studies, in which we let macaques move freely when grasping small objects, showed postural preferences for sitting with the elbow flexed and the torso bent. Post hoc visual inspection of our video recording indicated small humeral abduction limited to the parasagittal plane. In the present paper, we give a quantitative analysis of these differences for studies of both macaques and humans.

Non-human primates and humans differ also in their range of wrist motion. Macaques walk at the fingers, with the metacarpal hyperextend ('digitigrade' walk [24]). During stable stance, macaques place their hand sideways, making large excursion of the wrist in the ulnar direction. Morphological studies show that radial and ulnar abduction of the wrist is larger in cercopithecoid monkeys (e.g. Macaca maurus) than it is in humans (the whole range of radio-ulnar excursion from

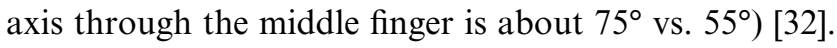
This element of postural behavior is considered to be significant for the biomechanics of the whole forelimb during diverse types of locomotion [28]. It is yet unknown how the greater range of wrist excursion in macaques affects the organization of prehension. 


\section{Study hypotheses}

In summary, all the above observations indicate that there are a number of biomechanical constraints specific to non-human primates and suggest that macaque's grasping must result from, at least in part, a different control mechanism than that in humans. For this study, we started with the hypothesis that these species' differences would result in different kinematic patterns, revealing instabilities of posture, such as large variations in the velocity and acceleration profiles. Given that goal-directed grasping produce similar kinematic across species, we investigated here the detailed course of reaching actions to determine where these different morphological constraints might demonstrate an effect.

As it is difficult to carry out systematic studies in unconstrained condition, little is known of how nonhuman primates organize quick natural prehension actions, and which postural adjustments they would make when allowed to move freely. In our study, we followed a protocol in which the animals were left free of their movements. They were ranging in a relatively large area $\left(250 \mathrm{~m}^{2}\right)$, which constantly allow to train muscles at a variety of substrates (rocks, wooden stems, sandy, grassy floors). We measured postural position preferences (quadruped stance, sitting or squat) for prehension and with the arm either flexed or stretched.

\section{Methods}

\subsection{Subjects}

We recorded the kinematics of arm movement in five human subjects and five macaques, performing the same task; namely, grasping a small object (raisin or peanut, $\sim 10 \times 10 \times 10 \mathrm{~mm}^{3}$ ) and bring it to the mouth (to eat it). Human participants were right-handed adults (two women, three men, age 25-38, $X=30.6 \pm$ 5.6; laterality quotient (LQ) according to Oldfield's [25] questionnaire; $X=86 \pm 9$ ). Monkeys were five subadult to adult pig-tailed macaques (Macaca nemestrina; $5-12$ years, $X=8.6 \pm 2.9$; LQ, according to the observational protocol; $X=63 \pm 10$ ). A LQ of 100 refers to full right and preference.

\subsection{Behavioral data}

The humans were sitting comfortably in front of a table. They were instructed to initiate the task with the hand at the mouth and to, then, successively (for more than ten consecutive reaches) grab and bring to the mouth one single food item. In order to produce conditions similar to that occurring in the macaques study, we asked the subject to alternate hand use for each trial, grasping the object either unimanually, with the left or right hand, or bimanually with both hands in antiphase. Subjects were presented with several food items scattered on a flat surface. Humans were reaching within an area of $20-30 \mathrm{~cm}$ alongside the parasagittal plane away and towards the center of the body (Fig. 1). The distance was adjusted for each individual, such that the subject would reach the closest objects with the elbow slightly flexed $\left(\sim 120^{\circ}\right)$ and the farthest ones with a completely stretched arm $\left(\sim 180^{\circ}\right)$. In order to reproduce the time constraints in the macaques study (macaques were acting quickly under group competi-
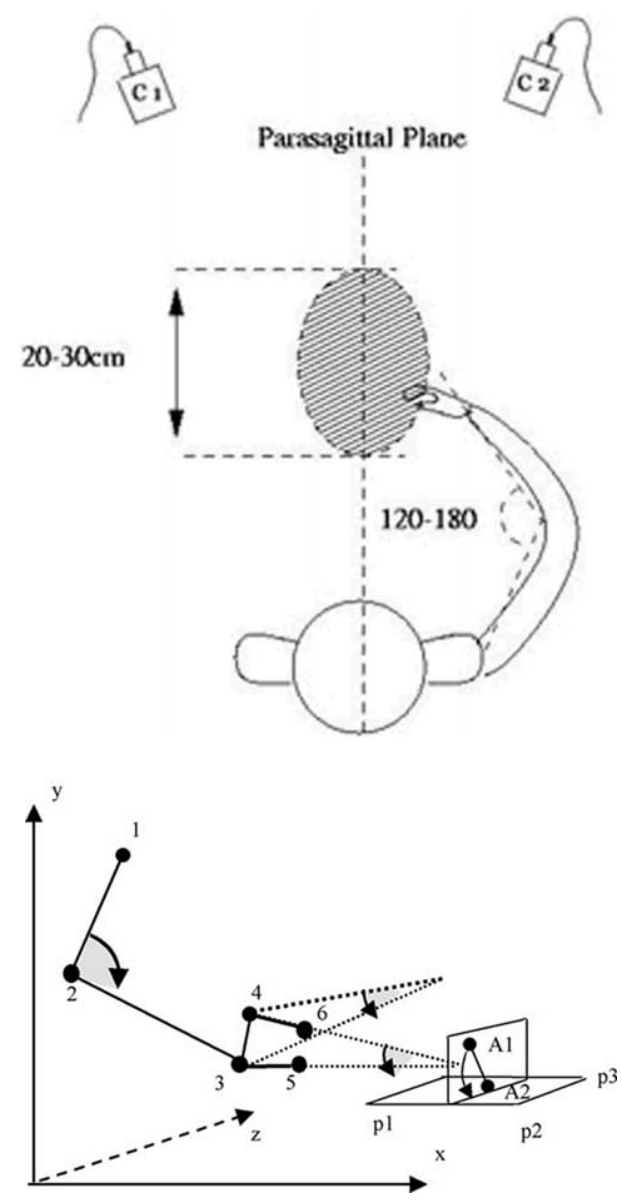

Fig. 1. Experimental set-up. Food items were scattered within an area of $20-30 \mathrm{~cm}$, alongside the parasagittal plane towards the center of the body, on a table in front of the subject (left figure), and on the floor in front of the monkey (Fig. 2). The distance from the body center to the food items was adjusted for each individual, such that the subject would reach the closest objects with the elbow slightly flexed $\left(\sim 120^{\circ}\right)$ and the farthest ones with a completely stretched arm $\left(\sim 180^{\circ}\right)$. The movements were recorded with two cameras $(\mathrm{C} 1, \mathrm{C} 2)$ placed at $45^{\circ}$ from the midsagittal plane and at a distance of at least $5 \mathrm{~m}$ (to avoid parallaxes). Referential of the hand in Cartesian space (right figure). Shoulder (1), elbow (2), wrist radial (3), wrist ulnar (4), thumb (5) and index finger (6). The elbow motion is measured by anatomical joint angle $(1-2-3)$. The wrist $(3-4)$ and the aperture of the fingers (5-6) are measured as segment angles relative to plane. The wrist and aperture points (A1 and A2) are perpendicular to a reference plane $(\mathrm{p} 1-\mathrm{p} 2-\mathrm{p} 3)$. 

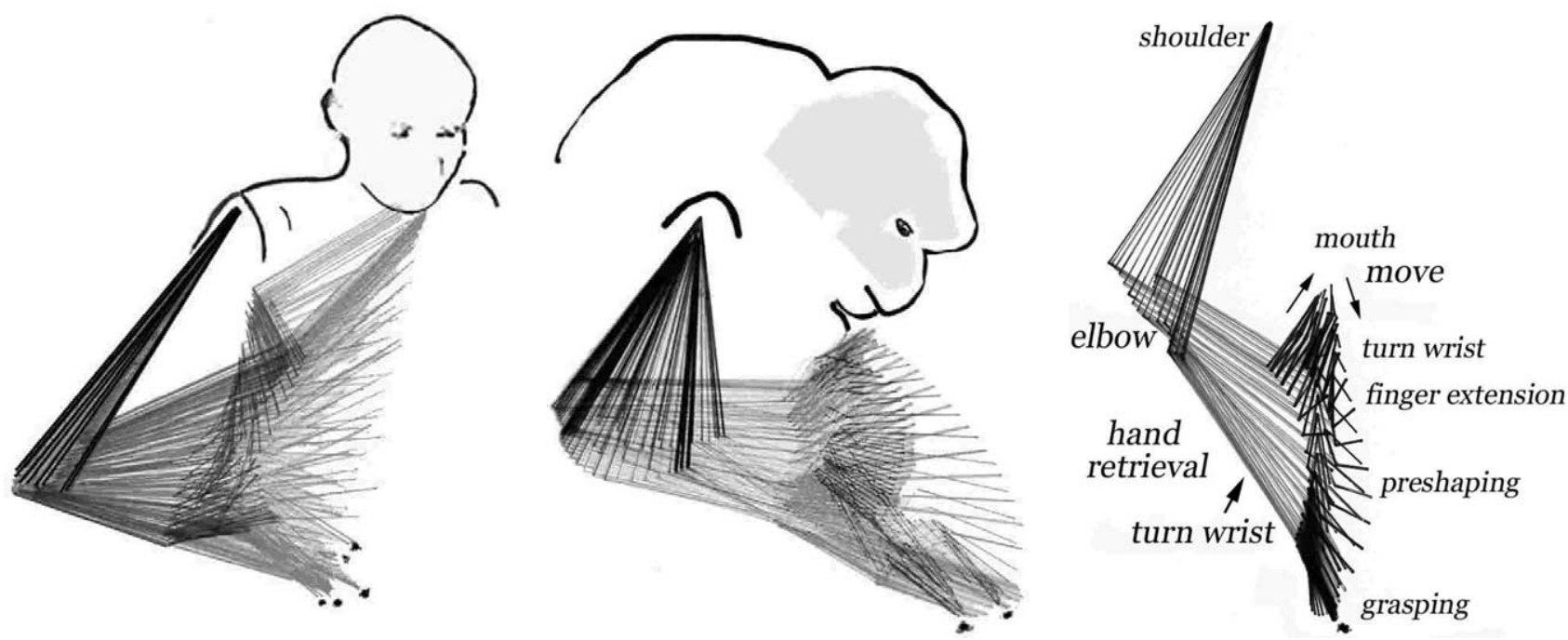

Fig. 2. Stick figures of prehension movements with the referential at the shoulder. Example of one human (left) performing five consecutive actions, and one monkey performing two actions (middle). Humans sat comfortably in front of a table. In this study, only reaching motions performed with a flexed arm's posture were analyzed. Note the larger elbow excursion in macaques compared to human. Stick figures of the seven-step sequence of prehension movement (right): step 1, move; step 2, turn wrist forward; step 3, extension; step 4, preshape; step 5, grasping; step 6, turn wrist backward; and step 7, retrieve. Monkeys were moving freely. Preferred posture corresponded to sitting with the elbow flexed and the torso bent forwards.
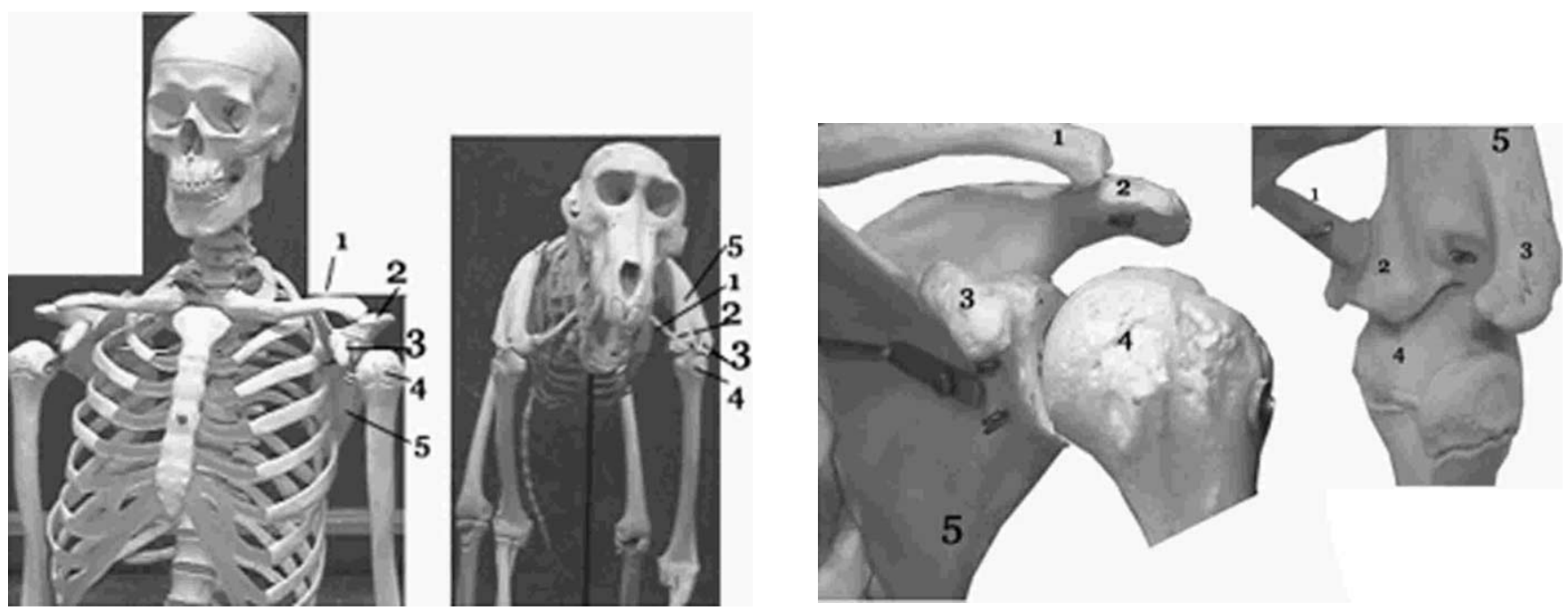

Fig. 3. The morphology of the shoulder joint differs importantly between species (left figure: humans; right figure: cercopithecoid monkeys). In cercopithecoid monkeys, the scapula (5) is in a lateral position (right figure); the acromion (2) and processus coracoideus (3) are large and robust and are inclined towards the head of the humerus (4); the humerus is shorter and has a stronger curvature at its dorsal aspect than human's. Clavicle (1).

tion), we set human subjects under time constraints, paced by a metronome ( $800 \mathrm{~ms} /$ cycle). Each trial was initiated by a 'go' signal. Motion of the two arms were recorded using two sets of six markers, one for each arm; the markers were placed on the shoulder joint (near acromion), on the elbow joint (near olecranon), on the ulnar and radial aspects of the wrist (near processus styloideus radii et ulnae), and on the thumb and index nails.

The macaques were studied in groups in their normal habitat (Berlin Zoo, Friedrichsfelde), a $250 \mathrm{~m}^{2}$ open air area of grass and sand. Macaques were performing very quickly grasping movements, using a precision grip. They preferred to sit slightly prone vs. squat or in a quadrupedal stance (Fig. 2). This postural preferences was in accordance with a flexed vs. a fully stretched arm's posture (according to the behavioral protocol, this observation is based on independent bouts of actions across several months; $X=75 \pm 8 \%$ ). For this study, we analyzed uniquely, the reaches with a flexed arm's posture in both species.

The kinematics of the macaque's and the human's prehension was reconstructed from 3D video images (using PEAK5 performance software, PAL $50 \mathrm{~Hz}$ ). We 

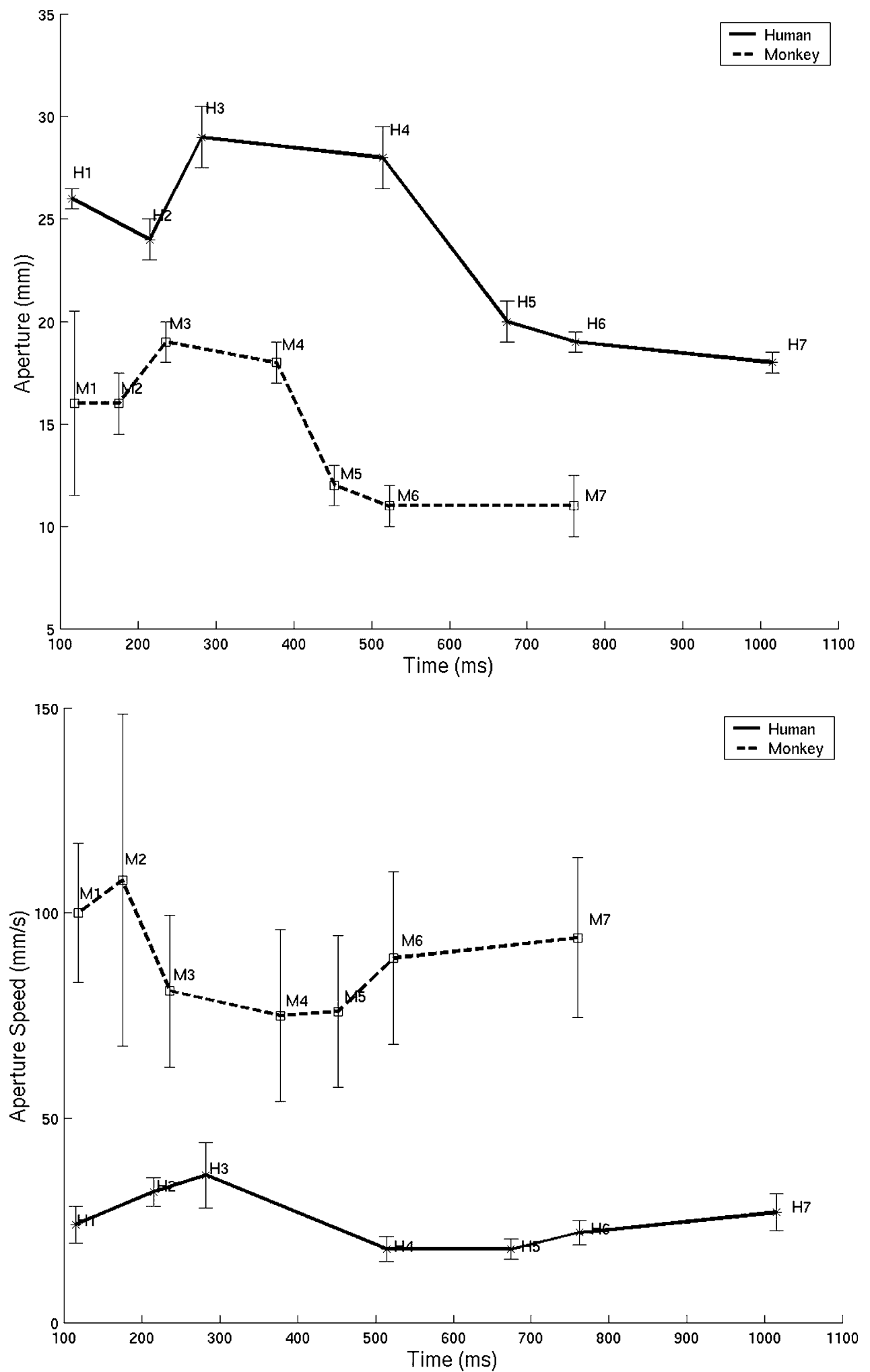

Fig. 4. Aperture width (top graph) within a seven-step sequence (ms). Monkeys (M) movements are faster than that of humans (H) throughout all the seven steps of the sequence. Aperture angle (thumb-forefinger distance) has the same biphasic course in both monkeys (M) and humans (H). Aperture is wider while the hand moves away from the mouth (step 1), fingers close more while the wrist rotates forwards (step 2), than fingers spread widely (step 3) and preshape (step 4), continuously closing for the grasp (step 5). With small aperture around the object, the wrist rotates backward (step 6) and the hand retrieves to the mouth (step 7). The angular velocity of the aperture is higher in monkeys than humans (middle graph). The angular velocity in monkey reaches a peak when the wrist rotates forwards (M2), while in humans it reaches its peak after the wrist rotation is complete and when the finger extension starts (H3). The angular acceleration (bottom graph) for the seven-step-sequence shows sharper starts and breaks in monkeys than it does in humans. Data are averages and S.D. across five subjects and ten actions per subject. 


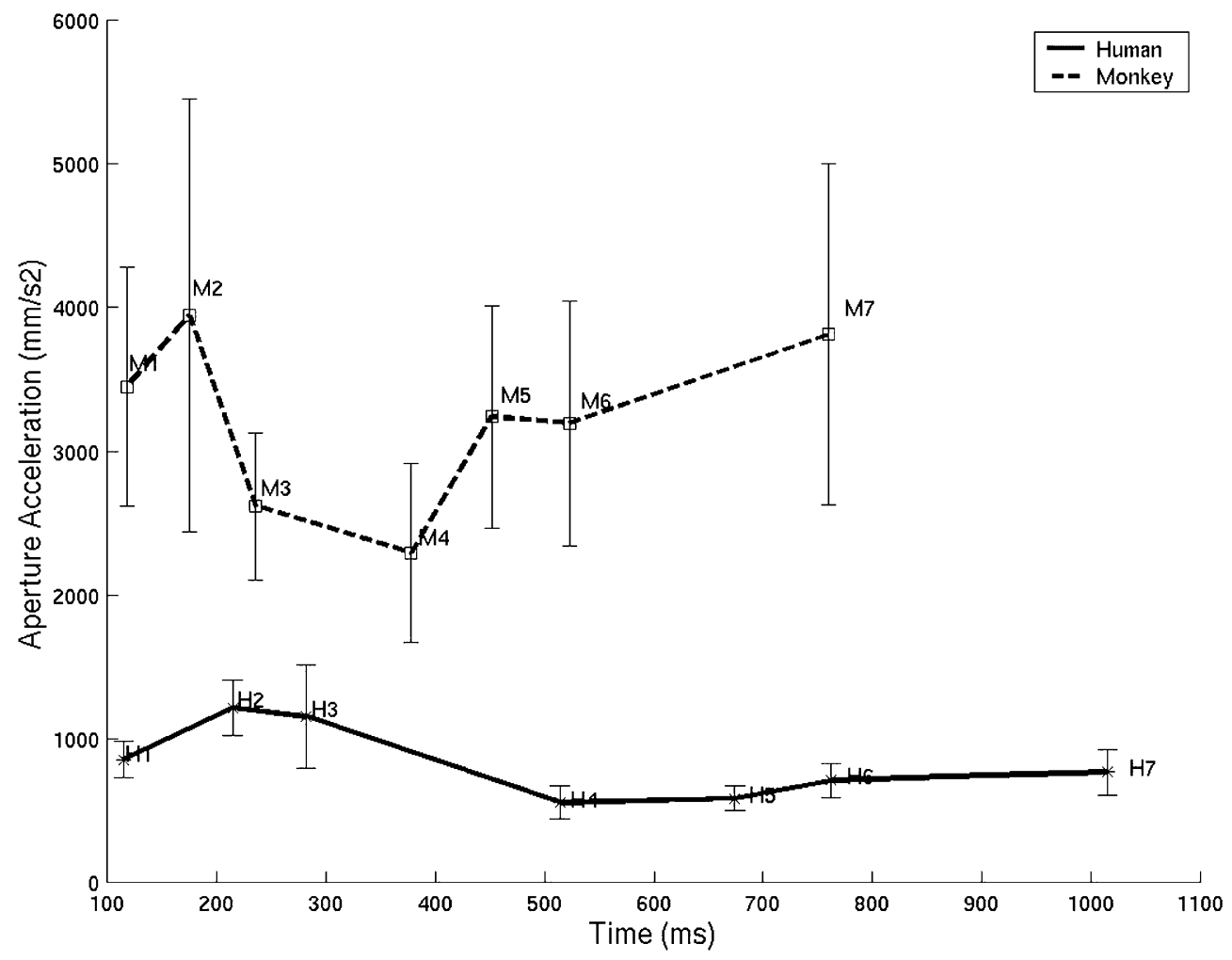

Fig. 4. (Continued)

filmed the macaques through a bullet-proof glass (40 $\mathrm{m}^{2}$; part of their wired enclosure) using two synchronized cameras. The area of filming was delimited using an industrial measuring cube (size $\sim 40 \times 40 \times 40 \mathrm{~cm}^{3}$; spatial accuracy $0.5 \mathrm{~mm}$ ). The food items were placed at the center of the filming area. Prior to testing, the macaques were trained to reach out through a wire, allowing us to stain white points at the shoulder (near the acromion) and elbow joints, and at the ulnar and radial joints of the wrist and on the fingers. The macaques, however, blurred the paint from their fingernails, but the glossy surface of the nails turned out to be reliable markers. The coloring of the fur served as rough measuring points. The exact positions of the joint were later reconstructed from the video recording by two observers delimiting manually the movement. The measurement was repeated until inter-observer correlation reached a verbal $100 \%$ agreement about locations of the points. For better comparison to the humans' study, recordings of the macaques movement were started when they held its hand at the mouth and end with the hand retrieved back to the mouth.

The 3D co-ordinates of the joint, with respect to a referential located on the shoulder, was reconstructed by linear translation using the PEAK 5 perform software. In Fig. 3, $X$-axis refers to the direction of the sagittosagittal plane (forward and backward motion); $Y$-axis is the sagitto-longitudinal plane (upward and downward motion) and $Z$-axis is alongside the sagitto- transversal plane (left and right motion). A second order Butterworth $10 \mathrm{~Hz}$ filter was applied on the data. Movements with the left hand were translated and rotated around the midsagittal plane and axis.

\subsection{Definition of submovements}

For each subject, we analyzed ten successful prehension cycles, i.e. a secure grip at the first approach. In humans, five with the right and five with the left hand, in macaques the hand that randomly was best in view to the camera (either right or left).

We defined seven-step sequence events during data scoring by jerks at the screen signaling changes in hand

Table 1

Correlation between unconstrained prehension kinematics as a percentage of the whole action cycle (including hand retrieval) comparing $M$. nemestrina vs. humans

Percentages of full cycle

$\begin{array}{lr}M . \text { nemestrina } & \\ \text { Elbow-wrist } & 2 \pm 1 \\ \text { Elbow-fingers } & 50 \pm 8 \\ \text { Wrist-finger } & 53 \pm 4 \\ \text { Humans } & \\ \text { Elbow-wrist } & 1 \pm 0.1 \\ \text { Elbow-fingers } & 41 \pm 4 \\ \text { Wrist-finger } & 45 \pm 1.1\end{array}$


and finger adjustments (Fig. 2). This was help to relate the data of single submovements to hand performances as following. The first jerk signaled (1) the movement onset of the fingers at the mouth, the next jerk signaled (2) the start of wrist rotation (pronation), until it was aligned. The wrist was aligned with (3) fully stretched fingers. After a short delay the thumb with a jerk opposed the forefinger in (4) the preshape. Preshaping phase was finished at (5) the first object contact of the fingertips. A jerk signaled when the finger (6) lift the objects. After a delay for retrieval (7) the wrist rotated backward (supination) and the full action cycle was accomplished by (8) the first mouth contact with the fingertips.

\subsection{Data evaluation}

A standard $t$-test (equivalent to a 1-way ANOVA) was applied to determine significance of the differences between the means of two independent samples, here macaques' and humans' data. Standard cross-correlation calculation was performed to measure the degree of cross-correlation across two joints in macaques' and humans' data.

\section{Results}

The general features of movement in macaques and humans are similar and follow a seven-step sequence: move, turn forward, extension, preshaping, grasping, turn backward, retrieve (Fig. 2).

Finger aperture in humans and macaques has the same biphasic course, i.e., a short and wide opening after peak velocity is followed by a slow closure phase (Fig. 4). Preshaping in both species has a very regular pattern with a quasi-constant time delay between elbow, wrist and finger aperture. Elbow and wrist motion is correlated with a time lag of about $1 \%$ of the reaching cycle (wrist starts with a slight delay). Elbow extension and finger aperture (preshape) is correlated with a time lag of $\approx 50 \%$ of the reaching cycle (Table 1). A $t$-test showed no significant difference between humans and macaques (significance level was set at 0.01 ): elbow-wrist: $P=0.25$, elbow-aperture: $P=$ 0.025 , wrist-aperture: $P=0.026$.

On average, macaques were reaching faster to the object than humans $(P=0.01)$, whereas they were as fast as humans during retrieval (Table 2). Faster motion during the reaching phase is achieved by faster rotation of the wrist and faster aperture of the fingers (steps for turn forwards, preshape and grasping). An ANOVA-nested design (two species, seven submovements) showed that the angular velocity and acceleration of the finger aperture and of the wrist were significantly higher in macaques than in humans (each
Table 2

Prehension kinematics ( $M$. nemestrina and humans); seven-step-segmented movements

\begin{tabular}{|c|c|c|c|}
\hline & M. nemestrina & Humans & $\begin{array}{l}P \text {-values } \\
(N 5 / 5)\end{array}$ \\
\hline Move (ms) & $118 \pm 32$ & $115 \pm 29$ & n.s. \\
\hline $\begin{array}{l}\text { Turn forwards } \\
(\mathrm{ms})\end{array}$ & $57 \pm 19$ & $100 \pm 30$ & 0.01 \\
\hline Extension (ms) & $61 \pm 14$ & $67 \pm 44$ & n.s. \\
\hline Preshaping (ms) & $142 \pm 46$ & $232 \pm 79$ & 0.01 \\
\hline Grasping (ms) & $74 \pm 13$ & $160 \pm 77$ & 0.02 \\
\hline Lift $(\mathrm{ms})$ & $71 \pm 13$ & $88 \pm 74$ & n.s. \\
\hline $\begin{array}{l}\text { Turn backwards } \\
\text { (ms) }\end{array}$ & $237 \pm 98$ & $253 \pm 95$ & n.s. \\
\hline \multirow{2}{*}{$\begin{array}{l}\text { Total reaching } \\
\text { duration }(\mathrm{ms})\end{array}$} & $378 \pm 28$ & $514 \pm 46$ & 0.001 \\
\hline & $\begin{array}{l}553 \pm 28^{\mathrm{a}} \\
350-500^{\mathrm{c}}\end{array}$ & $524 \pm 83^{b}$ & \\
\hline $\begin{array}{l}\text { Total retrieval } \\
\text { duration }(\mathrm{ms})\end{array}$ & $308 \pm 56$ & $341 \pm 85$ & n.s. \\
\hline Whole cycle (ms) & $760 \pm 34$ & $1015 \pm 61$ & 0.01 \\
\hline \multirow[t]{2}{*}{$\begin{array}{l}\text { Time to MGA } \\
(\mathrm{ms})\end{array}$} & $187 \pm 42$ & $212 \pm 36$ & 0.01 \\
\hline & & $321 \pm 91^{\mathrm{b}}$ & \\
\hline \multirow{2}{*}{$\begin{array}{l}\text { Maximum GA } \\
(\mathrm{mm})\end{array}$} & $35 \pm 5$ & $55 \pm 7$ & 0.01 \\
\hline & $27.5 \pm 0.03^{\mathrm{a}}$ & $80.5 \pm 11^{\mathrm{b}}$ & \\
\hline \multirow{2}{*}{$\begin{array}{l}\text { Time to MGA } \\
\text { ( } \% \text { of } \mathrm{MD})\end{array}$} & 49 & $41-$ & n.s. \\
\hline & $73-76^{\mathrm{a}}$ & $70^{\mathrm{b}}$ & \\
\hline
\end{tabular}

Movement duration (ms); values with \pm are S.D.; non-significant (n.s.).

${ }^{\text {a }}$ Prehension, M. mulatta, object size $1.5 \mathrm{~cm}$ at $20 \mathrm{~cm} \mathrm{[30].}$

${ }^{\mathrm{b}}$ Prehension, humans, object size $1.5 \mathrm{~cm}$ at $35 \mathrm{~cm} \mathrm{[26].}$

${ }^{\mathrm{c}}$ Pecking pigeons [20]; $t$-test.

Table 3

Prehension kinematics. $M$. nemestrina data vs. human data

\begin{tabular}{lccc}
\hline Kinematics & \multicolumn{1}{l}{ Elbow } & \multicolumn{1}{l}{ Wrist } & Aperture \\
\hline $\begin{array}{l}\text { Angular displacement } \\
\left({ }^{\circ}\right)\end{array}$ & $81 \pm 20$ & $37 \pm 19$ & $25 \pm 5$ \\
& $72 \pm 18$ & $36 \pm 17$ & $16 \pm 3$ \\
Angular speed $(\% / s)$ & $150 \pm 62$ & $230 \pm 102$ & $89 \pm 46$ \\
& $117 \pm 69$ & $176 \pm 118$ & $25 \pm 10$ \\
& $380^{\mathrm{a}}$ & $800^{\mathrm{a}}$ & \\
Angular peak velocity & $448 \pm 109$ & $737 \pm 156$ & $307 \pm 69$ \\
$(\% / \mathrm{s})$ & $290 \pm 38$ & $568 \pm 134$ & $93 \pm 25$ \\
& & & $237-290^{\mathrm{b}}$ \\
& $1772 \pm 694$ & $5443 \pm 3085$ & $3220 \pm 1894$ \\
Angular acceleration & & & \\
$\left(\% / \mathrm{s}^{2}\right)$ & $1003 \pm 424$ & $3370 \pm 2614$ & $824 \pm 420$ \\
\end{tabular}

\footnotetext{
a Prehension humans [19].

${ }^{\mathrm{b}}$ Beak opening pecking pigeons [20].
}

$P=0.01$; Table 3, Figs. 4 and 5). The elbow acceleration profile in macaques also showed significantly more variation than that in humans (Fig. 6). 

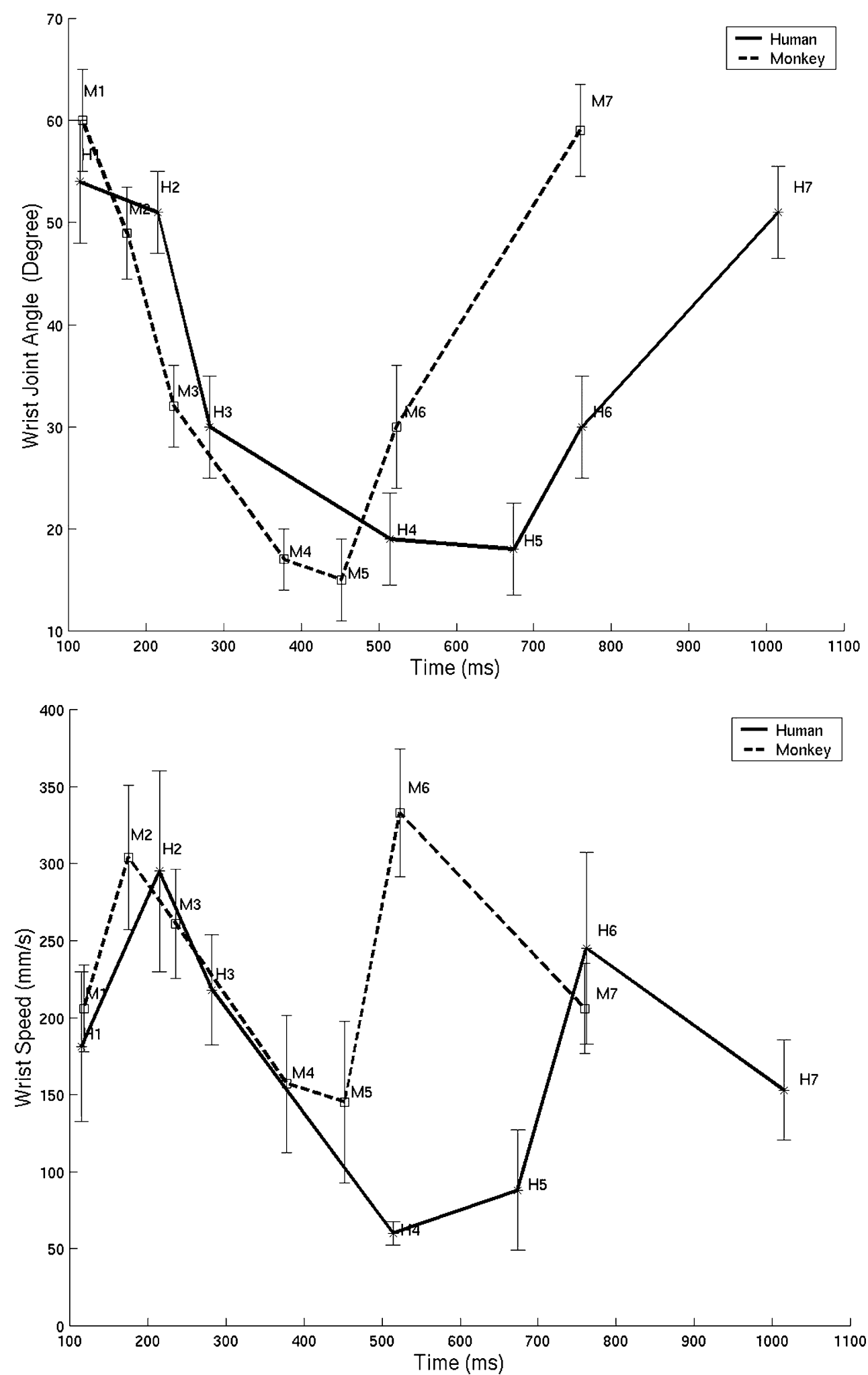

Fig. 5. Wrist joint angle excursion (top graph) within a seven-step sequence (ms). Both species have maximal angular excursion of the wrist when the hand moves away from the mouth (step 1) and minimal when the hand is aligned to the plane for grasping (step 5). Both species increases the wrist speed during forward rotation (step 2), and decreases during preshaping and grasping (step 4 and 5). Both increases wrist speed with backward rotation (step 6) and transport (step 7) to the mouth. The monkeys retrieve the hand with a higher speed, while both species in reaching have similar wrist speed (middle graph). Monkeys accelerate the wrist angle more strongly than humans during the motion of the hand away from the mouth (step 1; bottom graph). In humans, the acceleration of the wrist joint is maximal and as big as that in monkeys during forward rotation (step 2). Data are averages and S.D. across five subjects and ten actions per subject. 


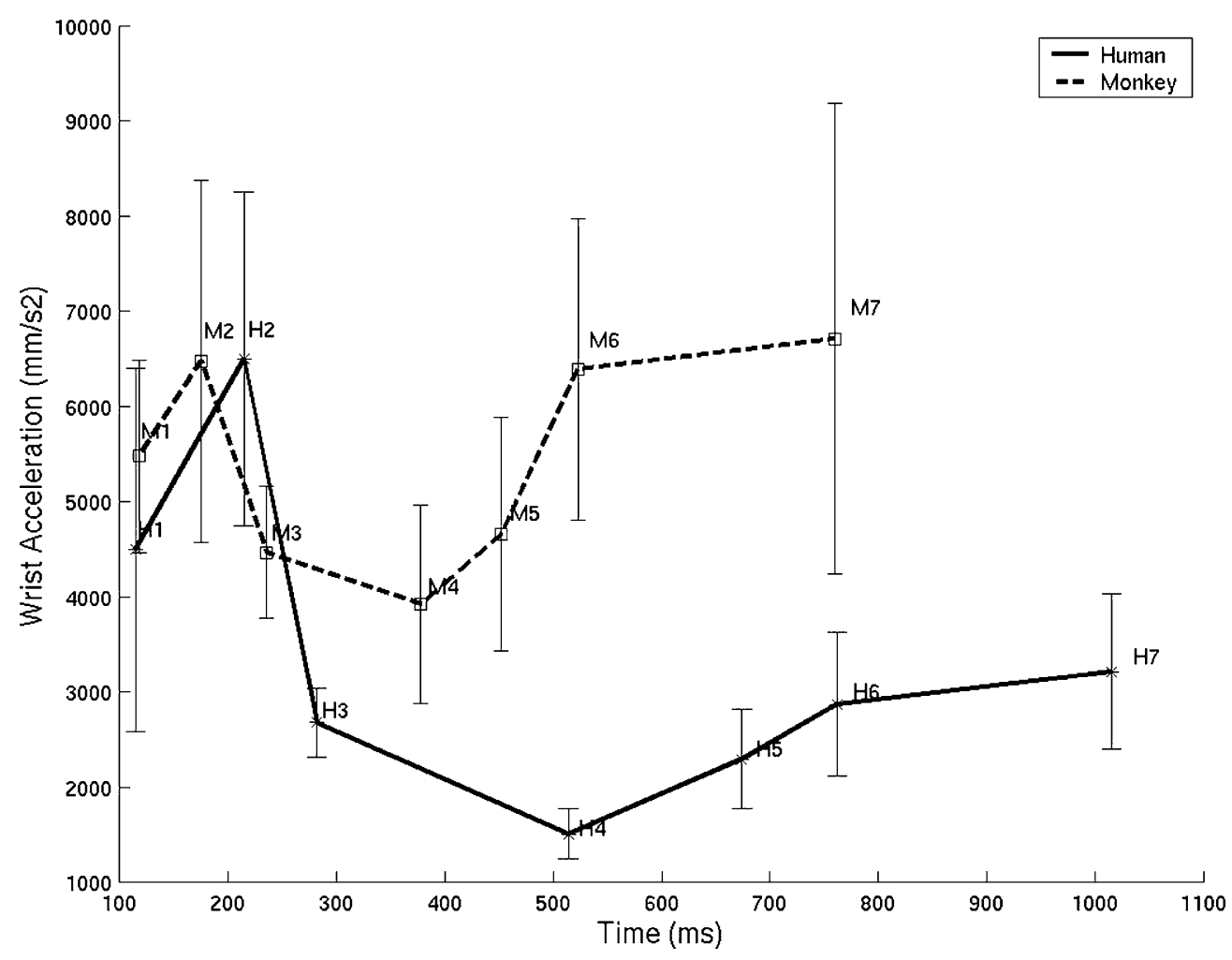

Fig. 5. (Continued)

Faster speed of movement in macaques is (partly at least) due to their having lighter limbs and greater muscular strength (relative to their body mass) than humans [8], as shown by simulation studies [4,5].

As mentioned earlier in the text, macaques shoulderjoint complex appears to allow less freedom of movements than does the human's one. Confirming this assumption, the natural prehension data show that, in contrast to humans, macaques make smaller shoulder abduction and wider elbow excursion through pronation (elbow excursion: $81 \pm 20^{\circ}$ vs. $72 \pm 18^{\circ}, t$-test: $P=0.01)$. Differences between species were striking alongside the horizontal plane (left and right motion, $Z$-axis). These were calculated as the ratio of arm length of species to the total displacement (humans $1.36 \pm 0.60$ against $0.68 \pm 0.24$ in macaques, $t$-test: $P=$ $0.001)$. While both did as large motion in the lateral plane (forward and backward, $X$-axis) as well as in the vertical plane (upward and downward, $Y$-axis; Table 4).
In addition, macaques made significantly larger movements of the torso than humans did (macaques: $36 \pm 24 \mathrm{~cm}$, humans: $15 \pm 5 \mathrm{~cm}$; $t$-test: $P=0.01$; Fig. 7). The larger motions of elbow and torso (bending towards the object) appear to compensate for the restricted motion of the shoulder in this study.

It is remarkable that, despite the larger instabilities of posture and joint kinematics in macaques, the hand paths in both species are similarly smooth (Fig. 8). This result confirms previous studies [20,30] (see Tables 2 and 3 for a comparison of the data) which show qualitatively and quantitatively similar trajectories for both effectors.

\section{Conclusions and perspectives}

We carried out a detailed comparative study of the kinematics of prehension in humans and macaques. In

Fig. 6. Elbow joint angle excursion (top graph) within a seven-step sequence (ms). Monkeys (M) make larger angular excursion of the elbow than humans $(\mathrm{H})$ within a shorter time period. The elbow extends continuously, while the hand moves away from the mouth (step 1), than the wrist rotates forwards (step 2), the fingers spread widely (step 3), and preshape for grasp (step 4). The elbow is at maximal extension as the fingers grasp the object (step 5), it flexes continuously while the wrist rotates backward (step 6) and the object is fed in the mouth (step 7). Both monkeys and humans start with similar angular velocity for the elbow joint (middle graph). Peak velocity is reached when the wrist rotates forward in both species (step 2). But at an earlier stage of the movement in monkeys than in humans. The angular velocity of the elbow in monkeys is higher during grasping (step 5) and hand retrieval (step 6 and 7). On average, the angular acceleration of the elbow joint is larger in monkeys. Both species decelerate elbow motion (bottom graph) during hand rotation (step 2). Monkeys accelerate elbow extension during finger preshaping and during object grasping (step 4 and 5), while humans decelerate during these phases. Conversely, humans accelerate strongly the elbow when they rotate the hand (step 3 and 6). Both species decelerate the elbow during the last hand retrieval phase to the mouth (step 7). Averages and S.D. across five subjects and for ten actions per subjects. 
agreement with the literature, we observe a number of qualitative features common to both species. These are (1) the timing of joint activity; the seven-step sequence and the relative phase of elbow/wrist/finger motion; (2) the smoothness of joint displacement and the bell-shape velocity profiles.

We, however, remark on a number of important differences concerning the organization of joint use. (1)
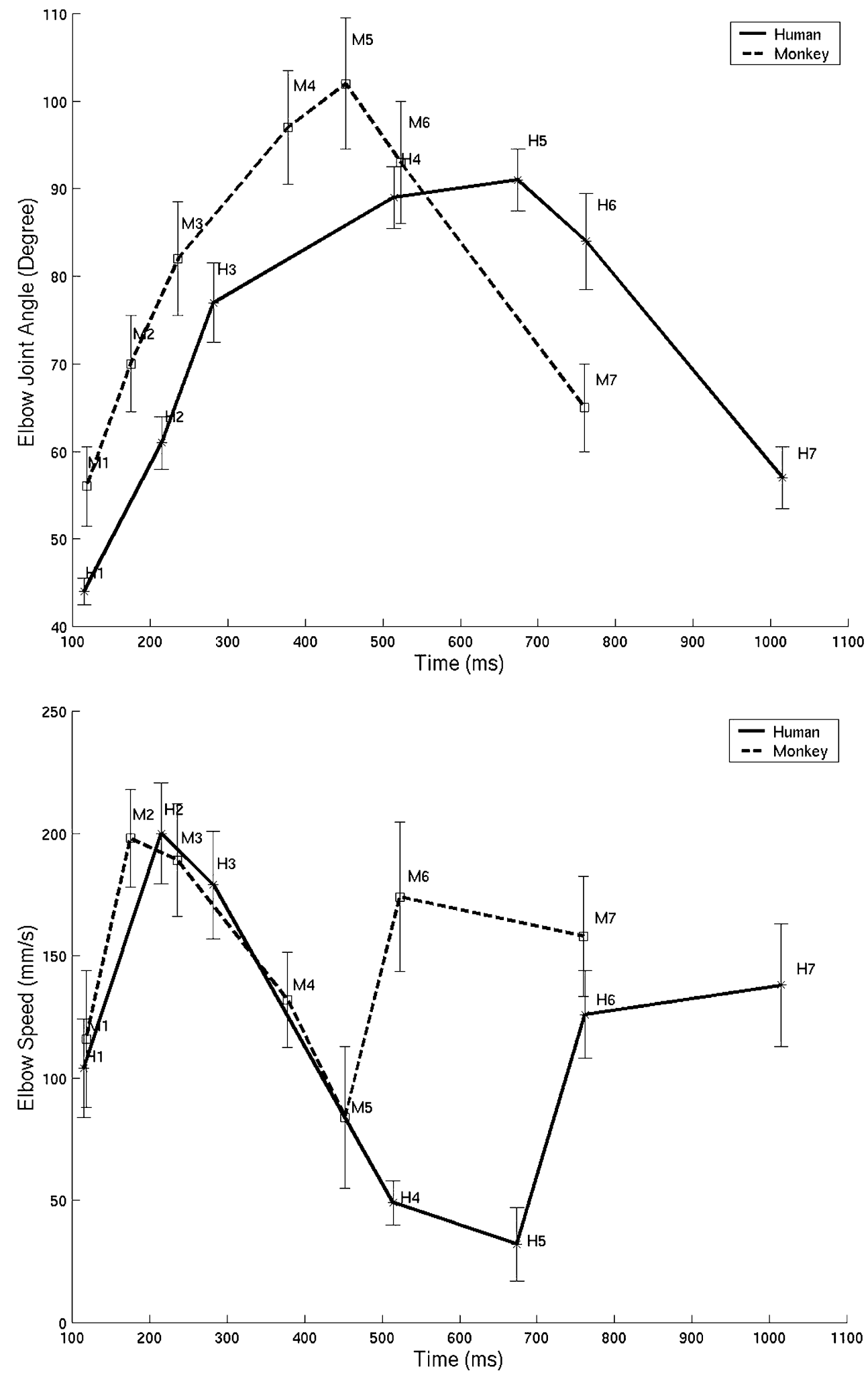

Fig. 6. 


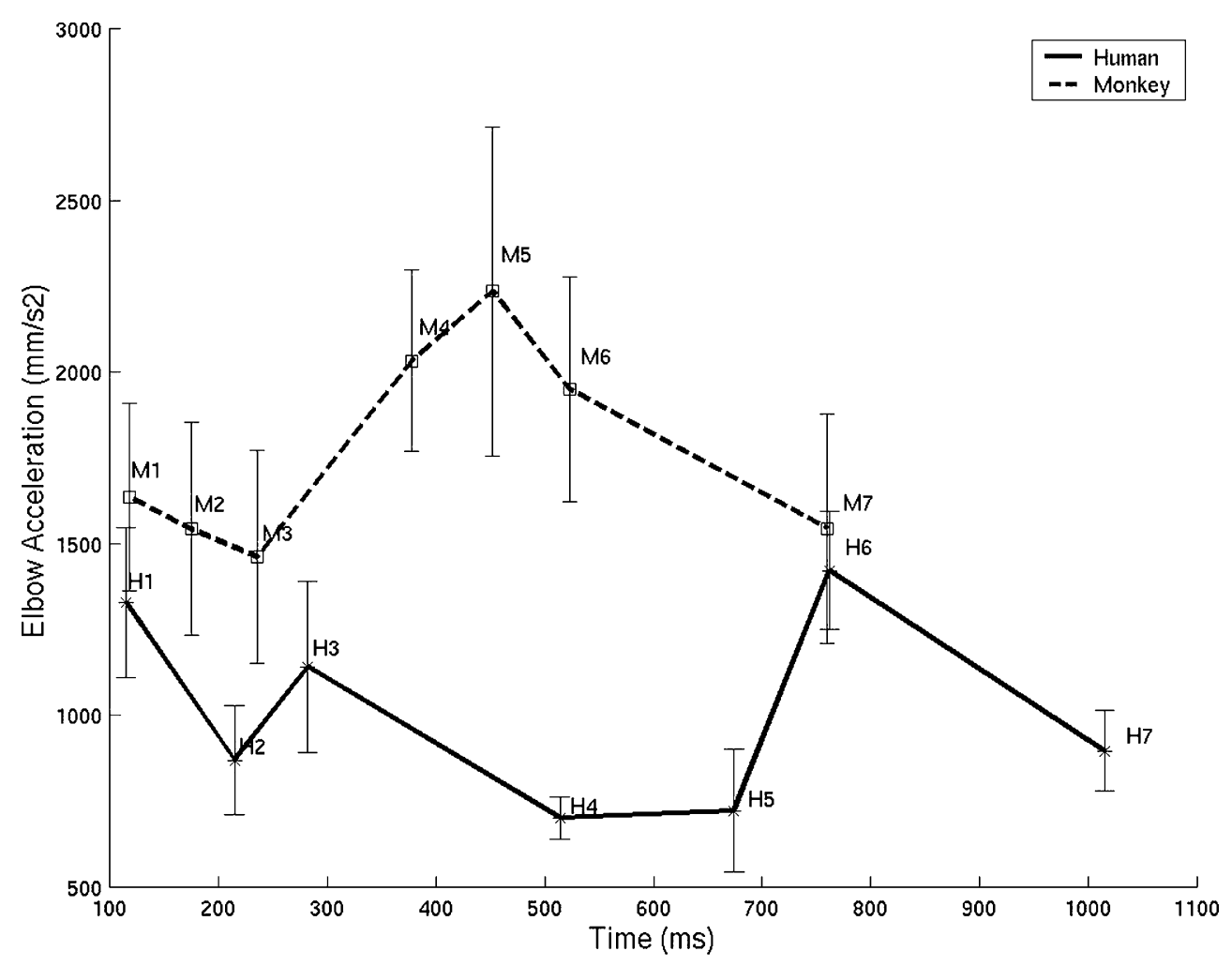

Fig. 6. (Continued)

Within a similar time frame, macaques produced significantly steeper and wider excursion of the elbow and of the wrist. Study of the velocity and acceleration profiles of the three-joint limb in macaques revealed stronger irregularities than in humans. (2) When performing the same task (grasping quickly and repetitively small objects within arm reach), macaques and humans chose opposite distributions of elbow and shoulder joints excursion: macaques preferred to make large elbow excursion and small shoulder abduction; in contrast, humans, made larger shoulder excursion and smaller elbow excursion.

Given that control and biomechanics are inter-linked (the control system being tuned to compensate for the biomechanical limitations), it is difficult to determine which of the biomechanics and of the control system is responsible for the above differences. We consider here four factors: (i) a different morphology of the shoulderjoint complex, (ii) a different muscular strength, (iii) a different posture, and (iv) a different control system.

(i) Macaque's and human's shoulder joints have important morphological differences, starting with the fact that macaques have their scapula in a lateral position. Comparative studies of the shoulder-joint morphology in macaques' and humans' skeletons suggest that macaques are limited in the range of humeral abduction they can make (because of the position of the acromion and coracoid above the head of humerus). In agreement with our data, laboratory experiments, in which the macaques' arm movements were not constrained by an external apparatus, showed that macaques made smaller shoulder excursion than humans when grasping for objects [34]. This hypothesis should, however, be tested against more data measuring the range of shoulder-joint motion (including scapula excursion) in freely behaving macaques and for other tasks than those reported in this paper. This biomechanical constraint can, however, not account completely for the limited shoulder abduction in our macaques' data, given that the extent of abduction required for the task at hand was contained within the range of possible shoulder excursion.

(ii) Macaques' arm muscles are hypertrophied compared to that of humans; a fact attributed to macaques' use of their forelimbs for locomotion [8]. Our data

Table 4

The relative displacement along each direction in monkeys and humans

\begin{tabular}{|c|c|c|c|c|c|}
\hline \multirow[t]{2}{*}{ Axis } & \multicolumn{2}{|c|}{ Humans } & \multicolumn{2}{|c|}{ Macaques } & \multirow[t]{2}{*}{$P$} \\
\hline & Ratio & S.D. & Ratio & S.D. & \\
\hline$X$ & 0.92 & 0.40 & 0.55 & 0.26 & 0.05 \\
\hline$Y$ & 0.78 & 0.19 & 0.65 & 0.18 & 0.2 \\
\hline$Z$ & 1.36 & 0.60 & 0.68 & 0.24 & 0.001 \\
\hline
\end{tabular}

Data are normalized by the total displacement; $t$-test: humans vs. macaques shoulder displacements. 

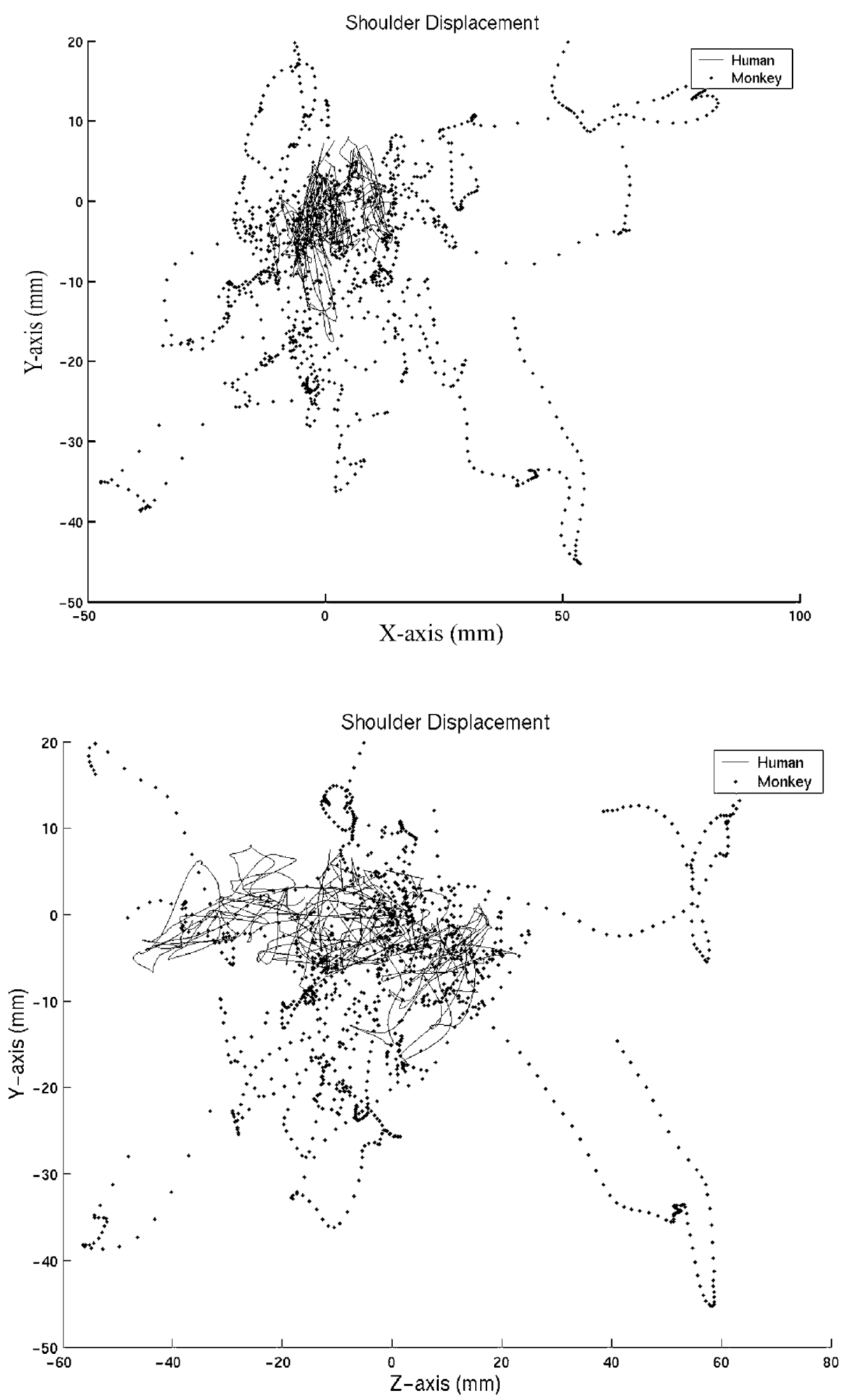

Fig. 7. Displacement of the torso (measured at the shoulder points) in Cartesian co-ordinates during prehension movements of five human subjects and five monkeys (each species 50 actions). The axes indicate the displacement in $\mathrm{mm}$ alongside the parasagittal plane (top graph) and on the horizontal plane (bottom graph). The monkeys make larger movements of the torso than humans. Both monkeys and humans were sitting during the grasping task. The large torso movements in monkeys compensated for smaller motions of the shoulder.

show that macaques control their muscles in a more demanding fashion, playing with sharp breaks and starts, while humans make smoother transitions of speed (possibly working more with co-contraction of muscles to constantly stabilize the arm). The stronger musculature in macaques might explain this observa- 

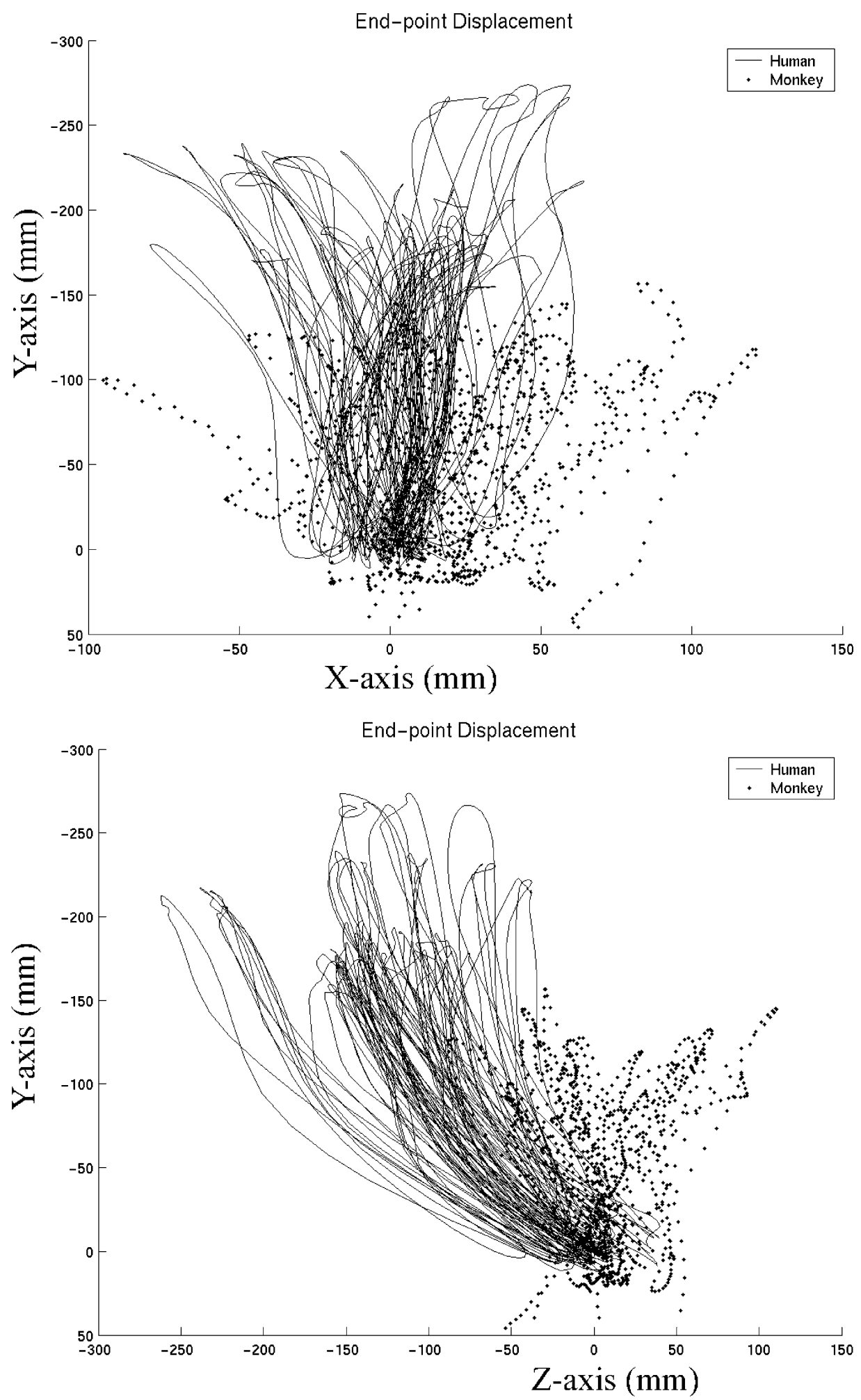

Fig. 8. Hand trajectory for prehension movements, performed with left and right hands by five human subjects and five monkeys (each species $n=50$ ). The hand starts at the mouth, then goes down to grasp and goes up to retrieve the object to the mouth. The loops at the top of the path indicate picking up of the object and hand retrieval. The axes indicate the displacement in $\mathrm{mm}$ alongside the parasagittal plane (top graph) and the horizontal plane (bottom graph). The trajectories are scaled to the length of the human and monkey arms. Monkey and human movements of the hand cover the same width of displacement (top graph). Note that the humans displaced hand path more largely into the horizontal plane (bottom graph). 
tion. Stronger muscles would produce bigger torque resulting in the large irregularities of acceleration profiles for the three-joint limb in our data.

(iii) In our study, macaques, who were free to adjust their posture, sat with the torso proned forward, supporting themselves with one hand. In contrast, the human subjects sat in front of a table, with the torso straight. It is possible that macaques' proned posture was unstable and, thus, required a different strategy to reduce potential instabilities. In order to better understand the effect of this factor, we will in future studies, conduct an experiment in which humans will reach in a crouched posture, similar to the macaques. Conversely, we will also conduct an experiment in which monkeys will be forced to sit with upright torso, similar to humans.

However, regardless of these postural differences, the overall plane of motion was similar in macaque and human experiments and both species produced similar end-point trajectories (as shown in Fig. 8). Moreover, the two experimental set-ups put similar constraints for the hand motion, as the distance object-mouth (when scaled to arm length) was the same for both monkeys and humans and was contained within the same overall space along the vertical and horizontal axes (Fig. 1).

In Ref. [4], we carried out computer simulations to reproduce the present experiments. Simulation meant to tell apart the part played by the physics in the differences observed in the two species behaviors. The simulations showed that it was physically possible for macaques to reach all objects by performing the same pattern of limb motion as humans did, and that such motions would not have resulted in a loss of equilibrium. This lead us to hypothesize that macaques chose a different co-ordinated pattern of the three-joint arm than humans, in our experiments, in order to achieve a similar range of comfort than humans did. Such a measure of comfort can be better understood, when considering each species' specific biomechanical constraints. As mentioned in Section 1, macaques have a smaller range of possible shoulder joint motion, while they have a bigger range of wrist motion. Acting like humans would have forced monkeys to use their shoulder joint close to its maximal range of motion, an unlikely strategy for achieving comfortable range of motion [13].

(iv) In point (i) above, we stressed the biomechanical differences in shoulder joint and their implications in constraining macaques' shoulder motion. This factor can, however, not account for the highly constrained (more constrained than made necessary by the biomechanics) movements of the macaques' shoulder in our data. Another reason, behind this behavior, could be that macaque's grasping results from a different (at least in part) control mechanism.

If such differences exist, it would be important to quantify their importance, as it would question the use of macaques' neurological study for reaching as a model of similar CNS control in humans. Given that macaques use their arms for locomotion, it is possible that the control system underlying macaque locomotion privileges motions in the parasagittal plane. However, in addition to directing the limbs for locomotion, macaques' control system must perform fine-tuned manipulation of objects. Macaques' CNS must thus have developed so as to cope with the possibly conflicting demands on hand function. In a view which favors hierarchical motor control (Section 1), the locomotor control system could be represented as a simple oscillatory circuit (CPG). Grasping behavior would be directed by higher motor centers, which build upon (and are partly biased by) the primitive patterns of locomotion. In future work, we will investigate the effect of using different models of primitive neural circuits for representing the above hypothetical differences between macaques and humans arm controls $[1,4,5]$.

\section{Acknowledgements}

This study was supported by the Deutsche Forschungsgemeinschaft (DFG CH 149/4-1) and in part by a grant of the Freie Universitaet (FU), Berlin (KFN, MCH 19/95-1) to Marianne Christel. Aude Billard is supported by a personal fellowship from the Swiss National Science Foundation. Part of the human data was acquired with optoelectronic devices at the Institute of Professor M. Jeannerod with his help and that of P.H. Weiss and Y. Paulignan. We like to thank them. We thank C. Niemitz, FU Berlin providing access to PEAK5 analysis and S. Bavar, Ch. Kambach and M. Morandi for help in data scoring. Our special thanks go to the stuff of the Zoo Friedrichsfelde. The first author likes to dedicate this paper to Holger Preuschoft, Professor emeritus, Department of Functional Morphology, RuhrUniversity Bochum, Germany.

\section{References}

[1] Arbib M, Billard A, Iacoboni M, Oztop E. Mirror neurons, imitation and (synthetic) brain imaging. Neural Networks 2000;13(8/9):953-73.

[2] Bermejo R, Zeigler HP. Trigeminal deafferentation and prehension in the pigeon. Behav Brain Res 1989;35(1):55-61.

[3] Bernstein NA. The coordination and regulation of movements. Oxford, UK: Pergamon Press, 1967.

[4] Billard A. Learning motor skills by imitation: a biologically inspired robotic model. Cybernet Syst 2001;32:1-2.

[5] Billard A, Christel MI, Schaal S. Technical Report 2001, Tech 01-366, Computer Science Department, University of Southern California.

[6] Bizzi W, Accornerro N, Chapple B, Hogan N. Posture control and trajectory formation during arm movement. J Neurosci 1984;4:2738-44.

[7] Bortoff GA, Strick PL. Corticospinal termination in two newworld primates: further evidence that corticomotoneuronal con- 
nections provide part of the neural substrate for manual dexterity. J Neurosci 1993;13(12):5105-18.

[8] Cheng EJ, Scott SH. Morphometry of Macaca mulatta forelimb. I. Shoulder and elbow muscles and segment inertial parameters. J Morphol 2000;245:206-24.

[9] Christel MI. Catarrhine primates grasping small objects-techniques and hand preferences. In: Anderson JR, Roeder JJ, Thierry B, Herrenschmidt N, editors. Curr Primatol, Behav Neurosci, Physiol and Reprod, vol. 3, Univ L Pasteur, Strasbourg. 1994;3:37-50.

[10] Christel MI, Fragaszy D. Manual function in Cebus apella. Digital mobility, preshaping, and endurance in repetitive grasping. Int J Primatol 2000;21(4):697-719.

[11] Fischer MS. Locomotory organs of mammals: new mechanics and feed-back pathways but conservative central control. Zoology 2001;103:230-9.

[12] Fitts PM. The information capacity of the human motor system in controlling the amplitude of the movement. Exp Psychol 1954;47:381-91.

[13] Gordon J. Receptors in muscle and their role in motor control. In: Downey \& Darling's physiological basis of rehabilitation medicine, 3rd ed. Boston: Butterworth \& Heinneman, 2001:81100.

[14] Heffner RS, Masterton RB. The role of the corticospinal tract in the evolution of human digital dexterity. Brain Behav Evol 1975;23:165-83.

[15] Hogan N. An organizing principle for a class of voluntary movements. J Neurosci 1984;4:2745-54.

[16] Howell AB, Strauss WL. The muscular system. In: Hartman CG, editor. Anatomy of the rhesus monkey. New York: Hafner, 1961:89-175.

[17] Illert M, Kümmel H. Reflex pathways from large muscle spindle afferents and recurrent axon collaterals to motoneurones of wrist and digit muscles: a comparison in cats, monkeys and humans. Exp Brain Res 1999;128:13-9.

[18] Jeannerod M. Intersegmental coordination during reaching at natural visual objects. In: Long J, Baddeley A, editors. Attention and performance. Hillsdale, NJ: Erlbaum, 1981:153-68.

[19] Jeannerod M. The formation of finger grip during prehension. A cortically mediated visuomotor pattern. Behav Brain Res 1986;19:99-116.

[20] Klein BG, Deich JD, Zeigler HP. Grasping in the pigeon (Columba livia): final common path mechanisms. Behav Brain Res 1985;18(3):201-13.

[21] Maier W. Vergleichende und funktionell-anatomische Untersuchungen an der Vorderextremität von Theropithecus gelada. Abhandlung der Senckenbergischen Naturforschenden Gesellschaft, Kramer Frankfurt, vol. 527, 1971.
[22] Martin JH, Cooper SE, Ghez C. Kinematic analysis of reaching in the cat. Exp Brain Res 1995;102:379-92.

[23] Nakajima K, Maier MA, Kirkwood PA, Lemon RN. Striking differences in transmission of corticospinal excitation to upper limb motoneurons in two primate species. J Neurophysiol 2000;84:698-709.

[24] Napier JR, Napier PA. Handbook of living primates. New York: Academic Press, 1967.

[25] Oldfield RC. The assessment and analysis of handedness: the Edinburgh inventory. Neuropsychologia 1979;97-113.

[26] Paulignan Y, MacKenzie C, Marteniuk R, Jeannerod M. Selective perturbation of visual input during prehension movements. Exp Brain Res 1991;83:502-12.

[27] Preuschoft H, Godinot M, Beard C, Nieschalk U, Jouffroy FK. Biomechanical considerations to explain important morphological characters of primate hands. In: Preuschoft H, Chivers DJ, editors. Hands of primates. New York: Springer, 1993:245-56.

[28] Preuschoft H, Günther MM, Christian M. A size dependence in prosimian locomotion and its implications for the distribution of body mass. Folia Primatol 1998;69(1):60-81.

[29] Rossignol S. Neural control of sterotypic limb movements. In: Handbook of physiology, vol. 12. New York: Oxford University Press, 1996:173-216.

[30] Roy AC, Paulignan Y, Farnè A, Jouffrais C, Boussaoud D. Hand kinematics during reaching and grasping in the macaque monkey. Behav Brain Res 2000;117:75-82.

[31] Schmidt M, Fischer MS. Cineradiographic study of forelimb movements during quadrupedal walking in the brown lemur (Eulemur fulvus, Primates: Lemuridae). Am J Phys Anthropol 2000;111(2):245-62.

[32] Schreiber H. Zur Morphologie der Primatenhand. I. Röntgenologische Untersuchungen an der Handwurzel der Affen. Anat Anzeiger 1934;78(20/24):369-429.

[33] Scott SH. Apparatus for measuring and perturbing shoulder and elbow joint positions and torques during reaching. J Neurosci Meth 1999;119-27.

[34] Scott SH, Kalaska JF. Reaching movements with similar paths but different arm orientations. I. Activity of individual cells in motor cortex. Am Physiol Soc 1997;826-52.

[35] Sergio LE, Scott SH. Hand and joint paths during reaching movements with and without vision. Exp Brain Res 1998; 122:157-64.

[36] Sonntag CF. On the anatomy of the Drill (Mandrillus leucophaeus). Proc Zool, London 1922;1:429-53.

[37] Stein PSG, Grillner S, Selverston AI, Stuart DG. Neurons, networks and motor behavior, a Bradford book. Cambridge, MA: MIT Press, 1997.

[38] Viviani P, Terzuolo C. Space-time invariance in learned motor skills. In: Stelmach GE, Requin J, editors. Tutorial in motor behavior. Amsterdam: North-Holland, 1980:525-33. 